\title{
Influence of Bias on the Friction Imaging of Ferroelectric Domains in Single Crystal Barium Titanate Energy Storage Materials
}

\author{
Lan Chen, ${ }^{1}$ Di Wu, ${ }^{1}$ Xiao-Min Xiong, ${ }^{1}$ Hai-Yan Wang, ${ }^{2}$ and Jin-Xiu Zhang ${ }^{1}$ \\ ${ }^{1}$ School of Physics and Engineering, Sun Yat-sen University, Guangzhou 510275, China \\ ${ }^{2}$ Guangzhou Institute of Measurement and Testing Technology, Guangzhou, Guangdong 510663, China \\ Correspondence should be addressed to Lan Chen; chenlan@mail.sysu.edu.cn and Xiao-Min Xiong; xiongxm@mail.sysu.edu.cn
}

Received 12 January 2014; Accepted 18 February 2014; Published 25 March 2014

Academic Editor: Wenjie Mai

Copyright (C) 2014 Lan Chen et al. This is an open access article distributed under the Creative Commons Attribution License, which permits unrestricted use, distribution, and reproduction in any medium, provided the original work is properly cited.

\begin{abstract}
The friction imaging of newlycleaved surface domains of single crystal $\mathrm{BaTiO}_{3}$ energy storage materials under both positive and negative voltage bias is investigated by scanning force microscope. When the bias was applied and reversed, three regions with different brightness and contrast in friction image indicated different response to the biases: the friction image of domain $\mathrm{A}$ displayed a great change in brightness while domains B and C displayed only a very small change. Possible mechanisms of the interesting phenomena originating from different static force between different charged tip and the periodical array of surface charges inside the inplane domains were proposed. These results provide a new method for the determination of the polarization direction for the domain parallel to the surface and may be useful in the investigation of ferroelectric energy storage materials, especially the relationship between the polarization direction of domain and the bias.
\end{abstract}

\section{Introduction}

Ferroelectric barium titanate ceramic $\left(\mathrm{BaTiO}_{3}\right)$ is a promising advanced material with the outstanding performance in dielectric properties for energy storage applications. With the high permittivity and volumetric efficiency $[1,2], \mathrm{BaTiO}_{3}$ was extensively used in electrical energy storage especially in supercapacitor and multilayer ceramic capacitors (MLCCs).

It is worth mentioning that the performance of the $\mathrm{BaTiO}_{3}$-based MLCCs was significantly affected by microstructures such as domain orientation, domain walls, and point defects $[3,4]$. The ferroelectric domain structure can be imaged by variety of techniques such as electron microscopy techniques, optical microscopy and $\mathrm{X}$-ray techniques, and scanning probe microscopy including piezoresponse force microscopy (PFM) and atomic force microscope (AFM) [5, 6]. Actually, friction mode of atomic force microscopy plays an important role in characterizing the surface of specimen by applying a friction force between tip and specimen [7-11].
Many previous works have reported the applications of friction imaging on characterizing the ferroelectric domain structure, which provided a clear picture for domain structures due to variant friction coefficients of different domains [12-14]. Recently, owing to the great importance of friction in industry, more and more research works were carried out to investigate the origin of friction in the micro- or even nanometer scale $[15,16]$ and even to explore efficient ways to control the friction force. Bluhm et al. [17] studied the friction imaging of domains with polarization perpendicular to the surface of specimen of guanidinium aluminum sulfate hexahydrate (GASH) and triglycine sulfate (TGA) and proposed that the chemical difference inside domains was the origin for the different friction coefficient, which could not be affected by the static electric force between tip and specimen. Park et al. [9] reported the possibility of making use of electric field to control the friction force between tip and semiconductor, although its exact mechanism is not clear until now. Although extensive attempts have been made in last decade, there is still no convincing explanation on 
the exact mechanism for different polarization domain with different friction coefficient.

In this report we investigate the friction imaging of newly cleaved surface of single crystal $\mathrm{BaTiO}_{3}$ under different voltage biases. It was found that different domains showed different dependence on voltage. Under different voltage biases, the friction imaging of a fraction of domains displayed a great change in brightness while that of the other domains displayed only a very small change. Possible mechanisms of the interesting phenomena originating from different static force between different charged tip and the periodical array of surface charges inside the inplane domains were proposed.

\section{Materials and Methods}

The multidomain single crystal $\mathrm{BaTiO}_{3}$ was prepared by the Institute of Physics, Chinese Academy of Sciences. First, the specimen was broken into two parts, one of which was about 1 millimeter thick. The newly formed cross section with the dimensions of $4 \mathrm{~mm} \times 1 \mathrm{~mm}$ was regarded as the newly cleaved surface. The newly cleaved surface was preferred because it was almost not affected by the pollutants and the free charges in air. The relative humidity was controlled to be $<40 \%$, and the experiments were carried out in about one hour.

Friction imaging was obtained through a commercial scanning force microscope SPA-300HV (Seiko, Japan). The scanning direction was perpendicular to the long beam of the cantilever Sil-AF01A. The cantilever with a resonant frequency of $12 \mathrm{kHz}$, a spring constant $k$ of $0.15 \mathrm{~N} / \mathrm{m}$, and a beam length of $400 \mu \mathrm{m}$ was used. The tip of the cantilever was coated by a gold film. During the experiment, the specimen stage was grounded and a voltage bias $(+12 \mathrm{~V}$ or $-12 \mathrm{~V})$ was applied onto the cantilever tip via a direct current voltage source.

The friction imaging was obtained on a same region of the newly cleaved surface under three different experimental conditions: without voltage bias, +12 voltage, and -12 voltage. The imaging region is about $20 \mu \mathrm{m} \times 20 \mu \mathrm{m}$ where there coexist many domains that could be imaged.

\section{Results and Discussions}

Figure 1 is the morphologic image and the friction images under different voltage biases. Three regions, respectively, marked A, B, and C in Figure 1, were discussed. From the morphologic image of Figure 1(a), it could be seen that the roughness of the cleaved surface was small. But the normal friction image without voltage bias (Figure 1(b)) has much contrast between three regions. In the normal friction image of Figure 1(b) without bias, Regions A and B showed almost the same brightness while Region $\mathrm{C}$ was darker than $\mathrm{A}$ and B. In addition, a significant fraction of domain boundaries was observed clearly. When $+12 \mathrm{~V}$ bias was applied, it was a surprise that the friction image in Figure 1(c) was much different from Figure 1(b). In Figure 1(c), Region A became darker, being a real difference from that in Figure 1(b).
However, the bright Region B still remained bright and the dark Region C remained dark in Figure 1(c). When $-12 \mathrm{~V}$ bias was applied, there was almost no change in the brightness of Regions B and C, whereas Region A changed back to bright (Figure 1(d)). That was to say, the friction images in Figures 1 (b) and $1(\mathrm{~d})$ were almost the same.

For single crystal materials, the regions with different brightness in friction image were sometimes considered as ferroelectric domains with different polarization directions $[18,19]$. Moreover, the cross section of single crystal multidomain $\mathrm{BaTiO}_{3}$ had many domains with different polarization directions including both perpendicular and parallel to the surface as reported by Soergel [20]. Therefore, it was suggested that the three regions in Figure 1 were associated with different oriented domains, which is similar to the results of PFM in [21]. In our experiment results, when the bias changed from $0 \mathrm{~V}$ to $+12 \mathrm{~V}$, domain $\mathrm{A}$ darkened while domain $\mathrm{B}$ remained bright. But while the bias changes into -12 voltage, domain A changed back into bright and domains B and C are kept the same. Three domains exhibiting different lateral force contrast were observed. The voltage bias had no effect on the friction force in domains $\mathrm{B}$ and $\mathrm{C}$ but had significant effect in domain $\mathrm{A}$. When the tip was applied to positive voltage, the friction force between positive charged tip and domain A was reduced dramatically compared with that for the negative or zero voltage bias. The dramatic change of friction force could be ascribed to the change of static electric interaction induced by the different charged tip.

Correia et al. [14] suggested that the static electric interaction could not change the friction force. Although they observed electric domains of GASH and TGS via scanning force microscope in the friction mode, what they concerned were the domains with the polarization direction perpendicular to the surface. However, in our experiments, we imaged the newly broken cross section of single multidomain $\mathrm{BaTiO}_{3}$ where there were all kinds of electric domains with different polarization directions scattered on the surface. The polarizations included not only the one perpendicular to the surface but also the parallel to the surface. It was the domains with polarization direction parallel to the surface that changed their brightness in the friction imaging under different voltage bias. According to the particular crystal structure of $\mathrm{BaTiO}_{3}$, it was inferred that the charge distribution on the surface of domains with polarization direction parallel to the surface was inhomogeneous, resulting in the friction force change under different voltage bias.

It was well known that $\mathrm{BaTiO}_{3}$ was tetragonal at room temperature, where $\mathrm{Ti}^{4+}$ was at the off-center position of the tetrahedral symmetry [1]. Thus the $\mathrm{Ti}^{4+}$ and the negative charge center were denoted as $\mathrm{O}^{2-}$ forming a permanent electrical dipole. Figure 2 schematically showed the alignment of the permanent electrical dipoles in the domain with the polarization direction parallel to the surface. As seen in Figure 2, the separation between $\mathrm{Ti}^{4+}$ and the negative charge center was $1.633 \AA$, much less than the separation $2.403 \AA$ between electric dipoles, which leads to an inhomogeneous charge distribution of surface. In friction imaging with scanning force microscope, while the tip was voltage biased, 


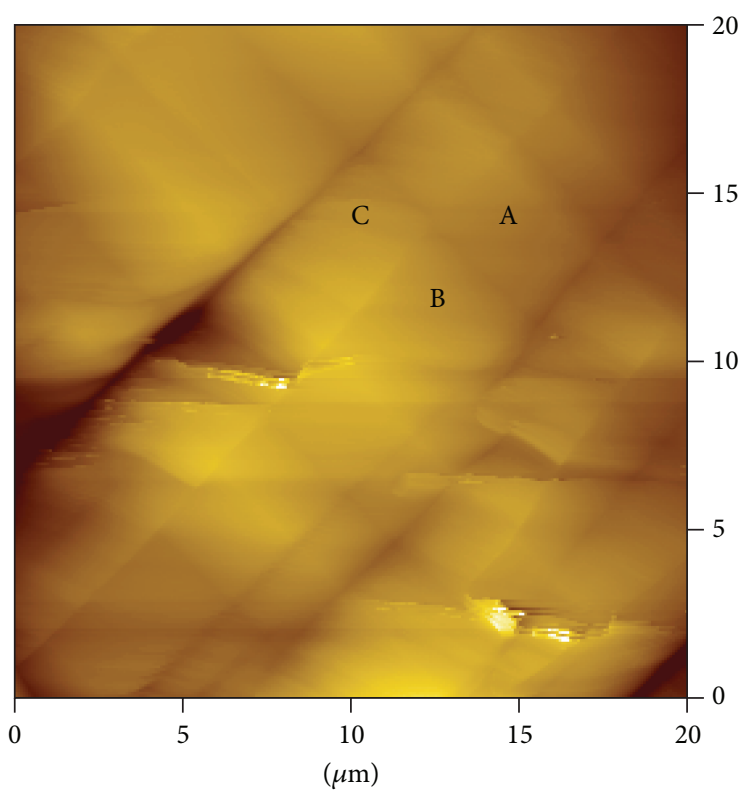

(a)

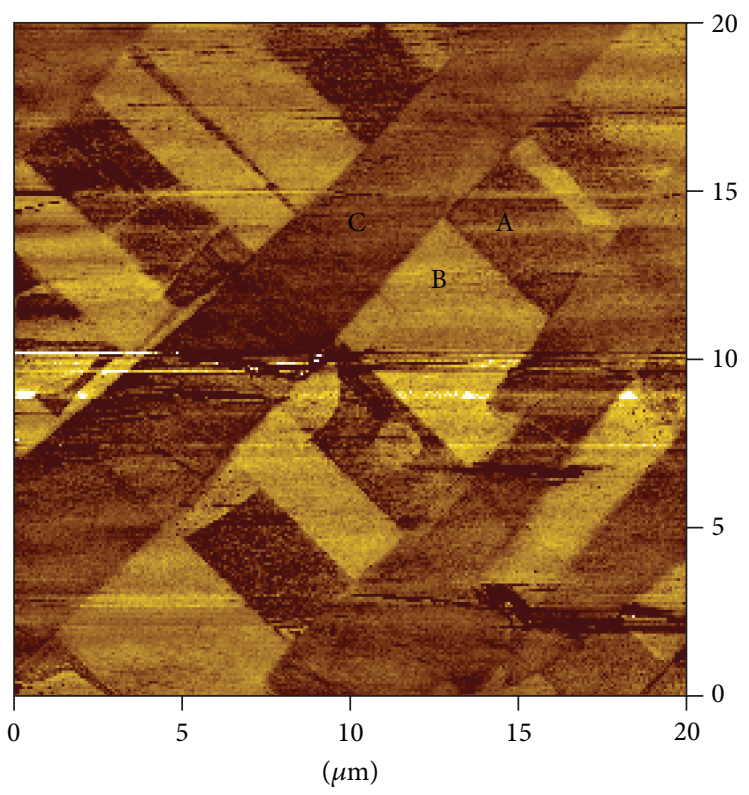

(c)

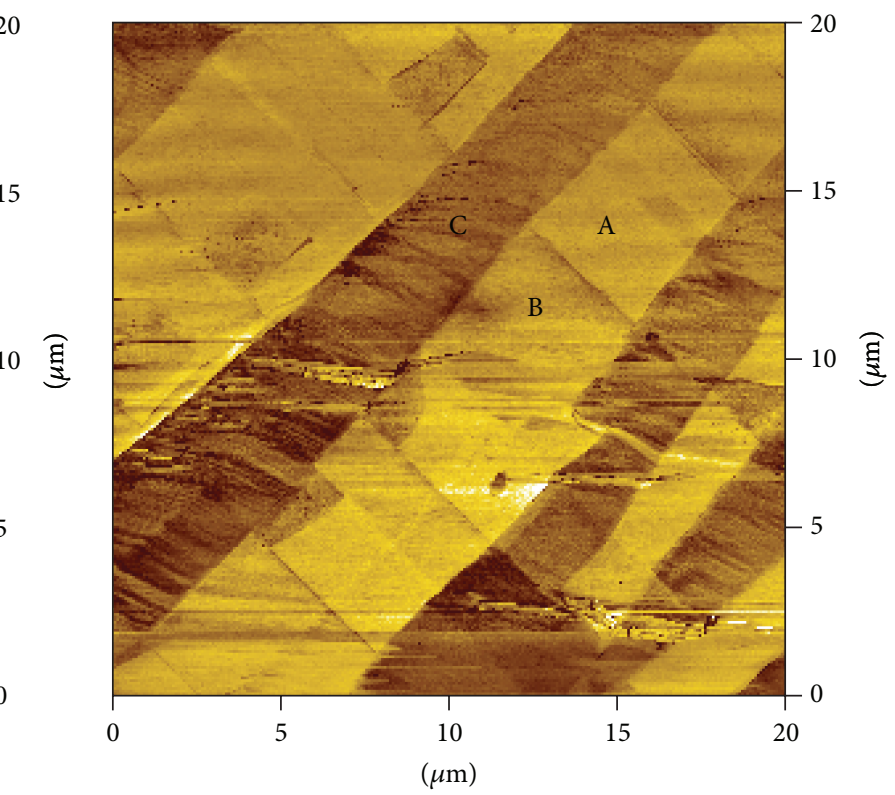

(b)

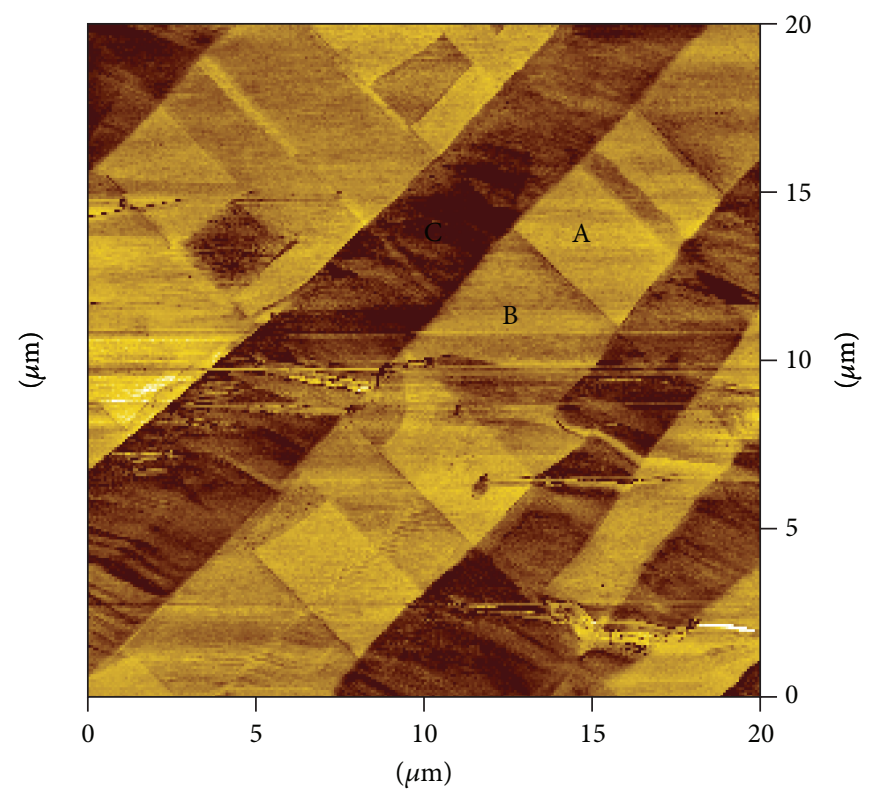

(d)

FIGURE 1: The morphologic image (a) and the friction images on a same region of the newly cleaved surface of single crystal multidomain $\mathrm{BaTiO}_{3}$ under three different experimental conditions: without voltage bias (b), +12 voltage (c), and -12 voltage (d). The specimen stage is grounded.

the tip was charged and the interaction between the charged tip and the inhomogeneous charge distribution resulted in an inhomogeneous energetic barrier distribution .

Different charged tip corresponds to different inhomogeneous energetic barrier distribution on the domain parallel to the surface, which is sketched as in Figure 3. For the case shown in Figure 3, it was assumed that the tip was scanned from left to right in the friction imaging. When the tip was positive voltage biased, the Coulomb interaction between the positive charged tip and the inhomogeneous charge distribution leaded to a zigzag energetic barrier distribution as in Figure 3(a). In this case, a small friction force was obtained. However, for the negative voltage bias, the situation was inversed. The zigzag energetic barrier was shown in Figure 3(b). A much large friction force was obtained. Additionally, when a certain angle existed between the scan direction and the polarization direction and a bias was applied to the tip, the friction force would change a little. In 


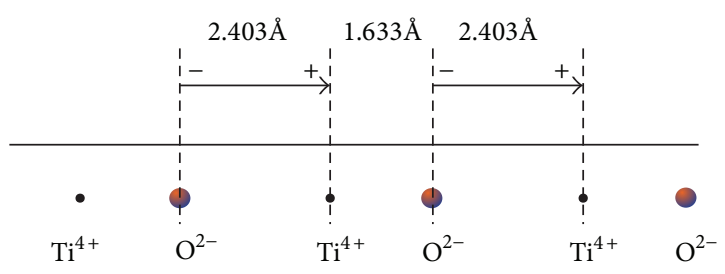

FIgURE 2: The alignment of the permanent electrical dipoles in the domain of $\mathrm{BaTiO}_{3}$ with the polarization direction parallel to the surface.
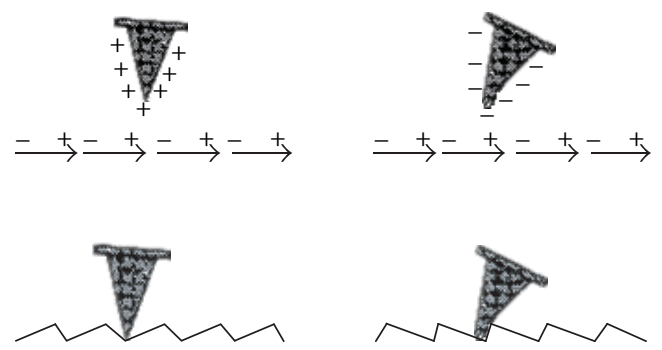

个Fiction force
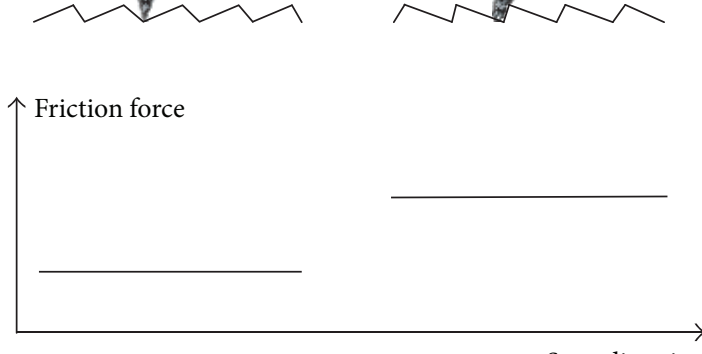

Scan direction

FIGURE 3: The friction force between the voltage-biased tip and the domain of $\mathrm{BaTiO}_{3}$ with the polarization direction parallel to the surface for the voltage bias is positive (left) and negative (right). It is assumed that the tip is scanned from left to right in the friction imaging. The Coulomb interaction between the charged tip and the inhomogeneous charge distribution of the domain leads to a zigzag energetic barrier distribution, which results in different friction force.

this case, the qualitative result was the same. At the same time the size of the tip had no effect on it.

Based on the above discussions and our experimental results, the brightness of the image for the domain A charged a lot: the friction force was much larger for the positive charged tip than that for the negative charged tip. It was thought that region A corresponded to the domain with polarization direction parallel to the surface and is almost the same as the scanning direction of the tip. As to the regions $\mathrm{B}$ and $\mathrm{C}$, the contrasts of them were not affected by the biases, and their boundaries well agreed with the morphologic image. So due to the titling of the domains, the polarization directions of domains $\mathrm{B}$ and $\mathrm{C}$ could not be judged only based on the friction force imaging and morphologic image and further investigations were necessary.

\section{Conclusions}

The newly cleaved surface of a single crystal multidomain $\mathrm{BaTiO}_{3}$ was investigated through the friction imaging with atomic force microscope. It was found that the brightness of some regions changed when the tip was differently voltage biased. It was proposed that the inhomogeneous charge distribution in the domain with polarization direction parallel to surface resulted in this change. The experimental results may provide a new method for the determination of the polarization direction for the domain parallel to the surface, which may be useful in the investigation of ferroelectric energy storage materials.

\section{Conflict of Interests}

The authors declare that there is no conflict of interests regarding the publication of this paper.

\section{Acknowledgments}

This investigation was supported by National Natural Science Foundation of China (NSFC) (Grant nos. 10974259, 11274391, and 11104357), Science and Technology Planning Project of Guangdong Province, China (Grant no. 2012B060100003), Fundamental Research Funds for the Central Universities (Grant nos. 121gpy36 and 09lgpy29), National Key Technology R\&D Program of the Ministry of Science and Technology (2011BAK15B04), and Science and Technology Planning Project of General Administration of Quality Supervision, Inspection and Quarantine of China (2011QK315).

\section{References}

[1] M. E. Lines and A. M. Glass, Principles and Applications of Ferroelectrics and Related Materials, Clarendon Press, Oxford, UK, 1997.

[2] W. Cai, C. Fu, Z. Lin, and X. Deng, "Vanadium doping effects on microstructure and dielectric properties of barium titanate ceramics," Ceramics International, vol. 37, no. 8, pp. 3643-3650, 2011.

[3] X. Ren, "Large electric-field-induced strain in ferroelectric crystals by point-defect-mediated reversible domain switching," Nature Materials, vol. 3, no. 2, pp. 91-94, 2004.

[4] M. Osada and T. Sasaki, "Two-dimensional dielectric nanosheets: novel nanoelectronics from nanocrystal building blocks," Advanced Materials, vol. 24, no. 2, pp. 210-228, 2012.

[5] E. Soergel, "Piezoresponse force microscopy (PFM)," Journal of Physics D: Applied Physics, vol. 44, no. 46, Article ID 464003, 2011.

[6] P. R. Potnis, N. T. Tsou, and J. E. Huber, "A review of domain modelling and domain imaging techniques in ferroelectric crystals," Materials, vol. 4, no. 2, pp. 417-447, 2011.

[7] K. S. K. Karuppiah, Y. Zhou, L. K. Woo, and S. Sundararajan, "Nanoscale friction switches: friction modulation of monomolecular assemblies using external electric fields," Langmuir, vol. 25, no. 20, pp. 12114-12119, 2009.

[8] B. Bhushan, K. J. Kwak, and M. Palacio, "Nanotribology and nanomechanics of AFM probe-based data recording technology," Journal of Physics: Condensed Matter, vol. 20, no. 36, Article ID 365207, 2008.

[9] J. Y. Park, D. F. Ogletree, P. A. Thiel, and M. Salmeron, "Electronic control of friction in silicon pn junctions," Science, vol. 313 , no. 5784 , p. 186, 2006. 
[10] J. Krim, "Friction at the atomic scale," Scientific American, vol. 275, no. 4, pp. 74-80, 1996.

[11] E. Gnecco, R. Bennewitz, T. Gyalog, and E. Meyer, "Friction experiments on the nanometre scale," Journal of Physics: Condensed Matter, vol. 13, no. 31, pp. R619-R642, 2001.

[12] R. Lüthi, H. Haefke, K. P. Meyer, E. Meyer, L. Howald, and H.J. Güntherodt, "Surface and domain structures of ferroelectric crystals studied with scanning force microscopy," Journal of Applied Physics, vol. 74, no. 12, pp. 7461-7471, 1993.

[13] H. Bluhm, U. D. Schwarz, K. P. Meyer, and R. Wiesendanger, "Anisotropy of sliding friction on the triglycine sulfate (010) surface," Applied Physics A: Materials Science \& Processing, vol. 61, no. 5, pp. 525-533, 1995.

[14] A. Correia, J. Massanell, N. García, A. P. Levanyuk, A. Zlatkin, and J. Przeslawski, "Friction force microscopy study of a cleaved ferroelectric surface: Time and temperature dependence of the contrast, evidence of domain structure branching," Applied Physics Letters, vol. 68, no. 20, pp. 2796-2798, 1996.

[15] A. Socoliuc, E. Gnecco, S. Maier et al., "Atomic-scale control of friction by actuation of nanometer-sized contacts," Science, vol. 313, no. 5784, pp. 207-210, 2006.

[16] R. W. Carpick, “Controlling friction,” Science, vol. 313, no. 5784, pp. 184-185, 2006.

[17] H. Bluhm, U. D. Schwarz, and R. Wiesendanger, "Origin of the ferroelectric domain contrast observed in lateral force microscopy," Physical Review B-Condensed Matter and Materials Physics, vol. 57, no. 1, pp. 161-169, 1998.

[18] R. W. Carpick, D. Y. Sasaki, and A. R. Burns, "Large friction anisotropy of a polydiacetylene monolayer," Tribology Letters, vol. 7, no. 2-3, pp. 79-85, 1999.

[19] C. Munuera, E. Barrena, and C. Ocal, "Deciphering structural domains of alkanethiol self-assembled configurations by friction force microscopy," Journal of Physical Chemistry A, vol. 111, no. 49, pp. 12721-12726, 2007.

[20] E. Soergel, "Visualization of ferroelectric domains in bulk single crystals," Applied Physics B: Lasers and Optics, vol. 81, no. 6, pp. 729-752, 2005.

[21] D. Liu, M. Chelf, and K. W. White, "Indentation plasticity of barium titanate single crystals: dislocation influence on ferroelectric domain walls," Acta Materialia, vol. 54, no. 17, pp. 4525-4531, 2006. 

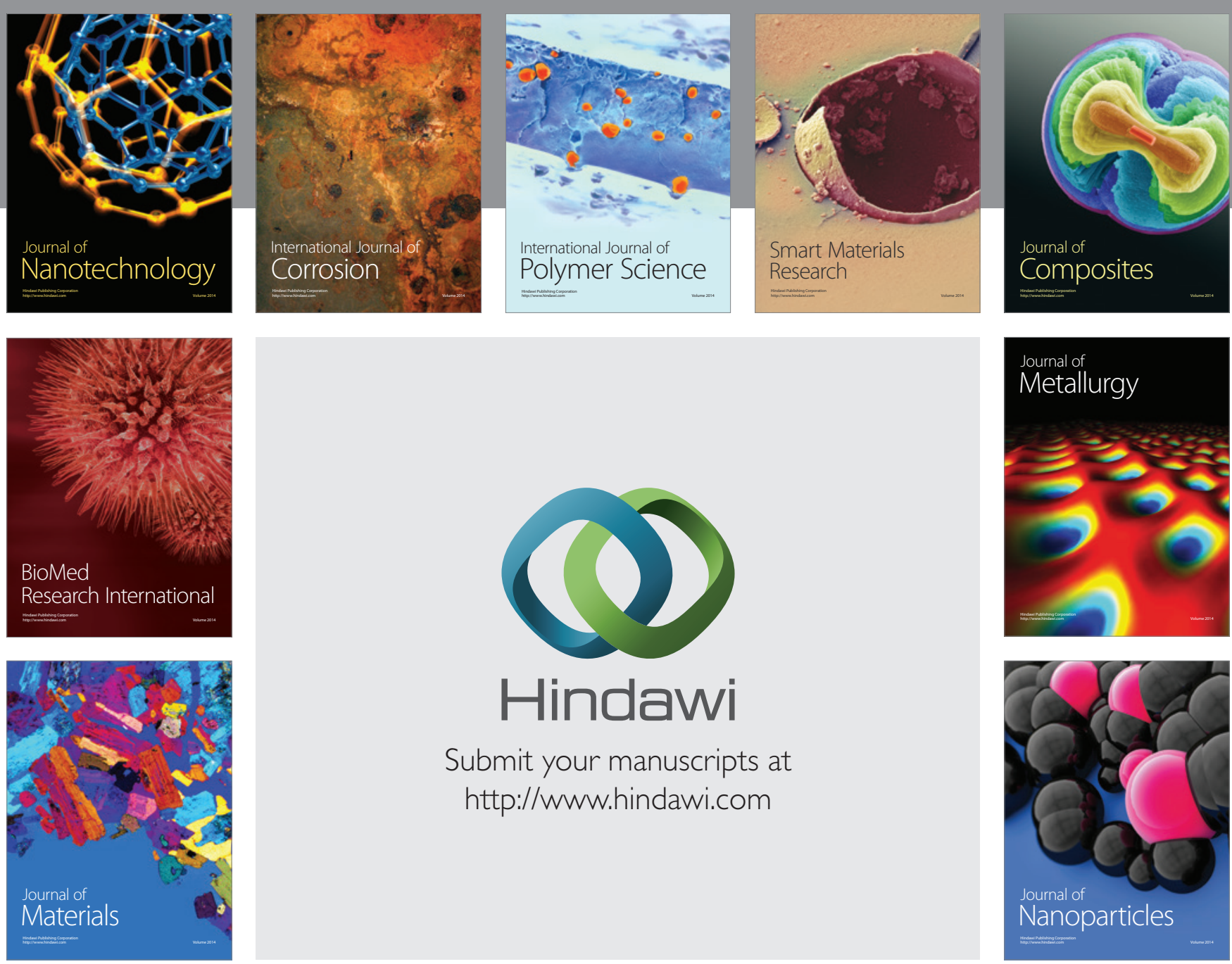

Submit your manuscripts at http://www.hindawi.com
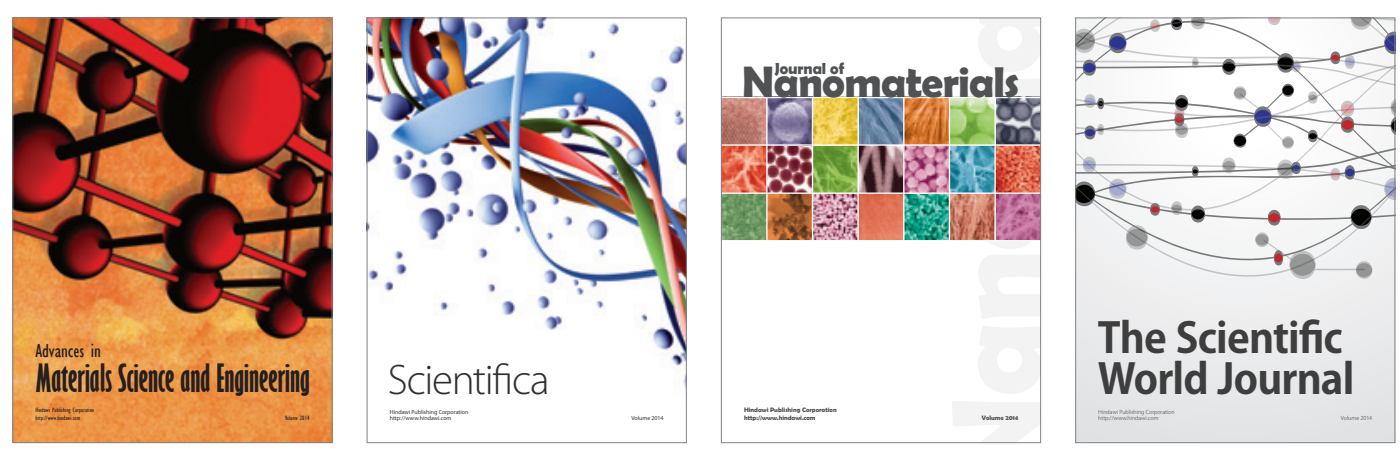

\section{The Scientific World Journal}
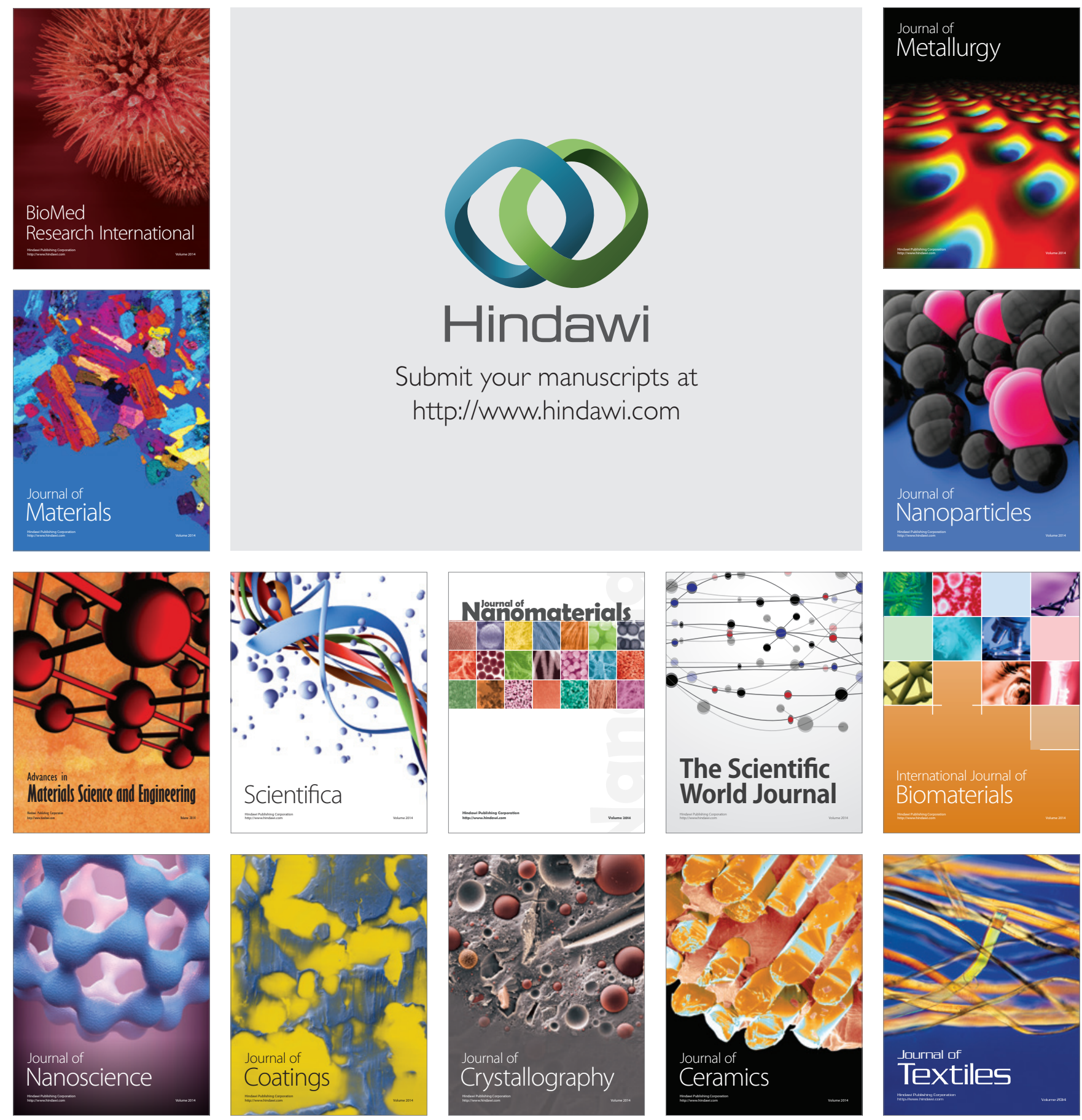